sphere. Either way, there must be a viscous drag from the asthenosphere which resists lithospheric motion.

On the face of it, active spreading seems unlikely, partly because the relatively thin lithosphere is required to transmit horizontal stresses over long distances and partly because of the difficulty in finding the force which produces the return flow in the asthenosphere. In addressing himself to the first of these problems Forristall notes McKenzie's point (Geophys. J., 18, 1; 1969) that tensile stresses are not required in the case where motion is caused by the descending lithosphere, but nevertheless uses them as a limiting condition in calculating the no-failure point in the lithospheric plate.

To be more specific, Forristall considers a model in which an elastic lithosphere, susceptible to failure according to the Mohr-Coulomb law, overlies a Newtonian asthenosphere. As the denser lithosphere descends into the less dense asthenosphere, the motion produces a relief of horizontal stress at the leading edge of the horizontal part of the plate and boundary stresses on the plate. What this stress relief means is that it is no longer valid to envisage the sinking plate pulling the rest of the lithosphere with it; it is the normal hydrostatic pressure at the trailing edge which pushes the plate towards the trench. Another consequence is that near the trench the horizontal principal stresses are lower than the vertical stresses.

In a series of stress calculations, Forristall then determines how great the stress relief can be without causing a failure in the plate. It turns out that, for horizontal stress relief at the leading edge of the horizontal part of the plate, the shear stress along the base of the lithosphere is constant. And because this shear stress arises from viscous drag, it can be related to the viscosity of the asthenosphere, given the vertical velocity gradient beneath the plate (actually calculated from the NavierStokes equation). What this means is that the no-failure condition for a lithosphere of any given thickness can be stated in terms of the maximum value of the asthenosphere's viscosity at any given asthenospheric thickness. Of course, the viscosity and thickness of the asthenosphere are not known with any certainty and so exact solutions are not possible. But by comparing his relationships with the viscosity and thickness values determined from the uplift of Fennoscandia (leaving aside the question of whether the behaviour of shield areas is really relevant to the suboceanic situation), Forristall concludes that to prevent lithospheric failure the asthenosphere must be less than 300 to $400 \mathrm{~km}$ thick; in other words, active spreading is only possible under this condition.
The problem of the driving force causing the return flow in the asthenosphere is less tractable and seems to require what really amounts to an ad $h o c$ solution and at least one ad hoc rationalization. It is easy enough to calculate from Forristall's analysis the value of the required pressure gradient, but less easy to discern its physical nature. Forristall postulates that the lithosphere thickens towards the trench, producing a horizontal pressure gradient beneath it. But a thickening of the lithosphere implies an excess mass and hence a large positive gravity anomaly. The observed gravity anomalies at trenches are certainly not large enough for Forristall's purpose, which leads him to speculate further that the excess mass is compensated by a mass deficiency lower in the mantle. One possi- bility here is that the asthenosphere extends to greater depths in trench regions.

As an example of the magnitudes involved, Forristall considers the lithospheric thickening necessary if the density contrast between the lithosphere and asthenosphere is $0.06 \mathrm{~g} \mathrm{~cm}^{-3}$. If the thickness $(x)$ of the lithosphere at the ridge is $20 \mathrm{~km}$ and the thickness $(y)$ of the asthenosphere (assumed constant) is $200 \mathrm{~km}$, the lithosphere must thicken to $180 \mathrm{~km}$ at the trench. For $x=50 \mathrm{~km}$ and $y=200 \mathrm{~km}$, the lithosphere must be $250 \mathrm{~km}$ thick at the trench. And if $x=20 \mathrm{~km}$ and $y=150$ $\mathrm{km}$, the plate must thicken to $95 \mathrm{~km}$. In other words, the required lithospheric thickening is far from trivial, although it is relatively smaller the thinner the asthenosphere.

\title{
Explaining the Spectra of Heavy Cosmic Rays
}

Four communications in next Monday's Nature Physical Science (January 29) discuss and supplement the evidence that there are relatively more heavy cosmic ray nuclei-iron for example -in the cosmic rays impinging on the Earth's atmosphere at energies of several tens of $\mathrm{GeV}$ per nucleon than at lower energies.

The situation is summarized in the diagram, which shows some of the data obtained by Ormes and Balasubrahmanyan as closed points. What they have done is to fly a balloon carrying detectors to measure particle charge and energy near the top of the atmosphere for $16 \mathrm{~h}$. The remainder of their data comprise the differential spectra of several nuclei and groups of nuclei at energies greater than $3.3 \mathrm{GeV}$ per nucleon. They conclude that the socalled secondary nuclei (that is those formed chiefly by the fragmentation of iron and nickel interacting with interstellar hydrogen) have somewhat steeper energy spectra than the cosmic ray "source" nuclei (chiefly iron and nickel). If these spectra continue to higher energies, say Ormes and Balasubrahmanyan, iron will become the most abundant nucleus apart from protons and helium nuclei at around 200 $\mathrm{GeV}$ per nucleon.

Although Ormes and Balasubrahmanyan draw some conclusions from their results, it is the other three groups of authors who delve deeply into the ramifications of the spectral differences between heavy and light nuclei for theories of the origin and acceleration of cosmic rays. Webber, Lezniak, Kish and Damle consider an equilibrium model for cosmic ray production and leakage from the Galaxy in which the "escape length" is assumed to be a function of energy. They find that a dependence of $E^{-1}$ fits the energy variations of both ratios $(\mathrm{C}+\mathrm{O}) /(\mathrm{Fe}+\mathrm{Ni})$ and (Nuclei with $Z=$ $17-25) /(\mathrm{Fe}+\mathrm{Ni})$. On the other hand, such a strong dependence on $E$ does not seem to be reflected by the ratio of the number of $\mathrm{Li}, \mathrm{Be}$ and $\mathrm{B}$ nuclei (thought to be secondary nuclei) to the number of $\mathrm{C}$ and $\mathrm{O}$ nuclei (primary nuclei). The question of the energy dependence of the theoretical term which describes the probability that galactic rays leave the Galaxy is also considered by Audouze and Cesarsky.

In the final communication Meneguzzi comes to the conclusion that the energy dependence of the abundance ratios is consistent with a decrease of the cosmic ray escape length from about $7 \mathrm{~g} \mathrm{~cm}^{-2}$ at about $2 \mathrm{GeV}$ per nucleon to less than $1 \mathrm{~g} \mathrm{~cm}^{-2}$ at 100 $\mathrm{GeV}$ per nucleon. $\mathrm{He}$ rejects the need for additional assumptions like a dependence of the mean free path on charge or a charged dependence of the spectral index of the source.

As Audouze and Cesarsky point out, many of the data now available seem to upset the theories that require cosmic rays to be confined to the disk of the Galaxy.

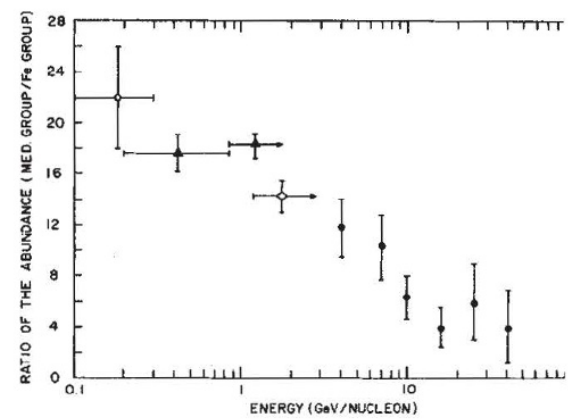

Ratio of abundance of "medium" nuclei $(6 \leqslant Z \leqslant 9)$ to those in the iron group $(23 \leqslant Z \leqslant 28)$ as a function of energy per nucleon. 